\title{
Caracterización del material compuesto de resina poliéster con partículas de caucho reciclado y su aplicabilidad en carrocerías.
}

\section{Characterization of the composite material made of polyester resin with particles of recycled rubber and its applicability in bodies.}

Ing. Segundo Manuel Espín-Lagos $\mathrm{Mg}^{1}$, Ing. Jorge Patricio Guamanquispe Toasa $\mathrm{Mg}^{2}$, Egda. Carolina Nataly Acosta Benavides ${ }^{3}$, Mayor. Anibal Lenin Jara-Olmedo ${ }^{4}$, Capt. Gustavo Vladimir León Castillo ${ }^{5}$, Ing. Martha Esperanza Sevilla Abarca $\mathrm{Mg}^{6}$.

Recibido: 13-12-2017 / Revisado: 01-02-2018 Aceptado: 02-03-2018/ Publicado: 01-04-2018

\begin{abstract}
.
The study of polymeric matrix composite every day increases its importance in the automotive and bodywork industry for its benefits such as low cost, new properties and easy manufacturing. The objective of this research was to obtain a polyester resin matrix composite reinforced with rubber particles from recycled tires with three different granule sizes varying its volumetric fraction in $10 \%, 20 \%, 30 \%, 35 \%$ and $40 \%$. The results show that tensile strength, flexion and compression of composite materials decrease with increasing rubber particle content, as well as tensile modulus tends to decrease with an increasing particle contents and a decreasing size of particle contents, while the elasticity module to flexion in the volumetric concentration of $10 \%$, evidences an increase of $84.11 \%$ for $\mathrm{G} 1 ; 91.16 \%$ for $\mathrm{G} 2$, and $155.93 \%$ for $\mathrm{G} 3$ compared to pure polyester resin (without reinforcement), therefore, it is also determined that it increases its flexural modulus of elasticity if the particle size is higher. With the mechanical properties obtained through the tests, it is determined

\footnotetext{
${ }^{1}$ Universidad Técnica de Ambato, Ecuador, sespin@uta.edu.ec

${ }^{2}$ Universidad Técnica de Ambato, Ecuador,jp.guamanquispe@uta.edu.ec

${ }^{3}$ Universidad Técnica de Ambato, Ecuador, cacosta0908@uta.edu.ec

${ }^{4}$ Centro de Investigación y Desarrollo de la Fuerza Aérea Ecuatoriana, lenin.jara@cidfae.gob.ec

${ }^{5}$ Centro de Investigación y Desarrollo de la Fuerza Aérea Ecuatoriana, gleon@ fae.mil.ec

${ }^{6}$ Universidad Técnica de Ambato, Ecuador, marthaesevilla@uta.edu.ec
}

DOI: https://doi.org/10.33262/cienciadigital.v2i2.72 
that the composite with G3 granule size is suitable for bending work since it increases its elasticity modulus turning it into an elastic material, and it would be used in exterior body parts such as fenders, dust covers, in bases between chassis and body to absorb vibrations, front and rear fender protectors to absorb impacts, in masks and as insulating material of noise, heat and electricity.

Keywords: Composite, rubber particles, recycled tires, mechanical properties, bodywork.

\section{Resumen.}

El estudio de materiales compuestos de matriz polimérica cada día incrementa su importancia en la industria automotriz y carrocera por sus beneficios como bajo costo, nuevas propiedades y facilidad de manufacturación. El objetivo de esta investigación fue obtener un material compuesto de matriz resina poliéster reforzado con partículas de caucho de neumáticos reciclados con tres granulometrías diferentes variando su fracción volumétrica de $10 \%, 20 \%, 30 \%, 35 \%$ y $40 \%$. Los resultados evidencian que la resistencia a tracción, flexión y compresión de los materiales compuestos disminuyen al aumentar el contenido de partículas de caucho, así como también el módulo de elasticidad a tracción tiende a disminuir al aumentar el contenido de las partículas y al disminuir el tamaño de partícula, mientras que el módulo de elasticidad a flexión en la concentración volumétrica de $10 \%$, se evidencia un incremento del $84,11 \%$ para G1; $91,16 \%$ para G2, y 155,93\% para G3 comparado con la resina poliéster pura (sin refuerzo), por lo tanto, también se determina que aumenta su módulo de elasticidad a flexión si mayor es el tamaño de la partícula. Con las propiedades mecánicas obtenidas mediante los ensayos, se determina que el material compuesto con granulometría G3 es apto para trabajar a flexión ya que incrementa su módulo de elasticidad convirtiéndole en un material elástico, y se utilizaría en partes exteriores de carrocerías como en guardafangos, guardapolvos, en bases entre chasis y carrocería para absorber vibraciones, en defensas de guardachoques delantero y posterior para absorber impactos y en masacrillas.

Palabras Claves: Material compuesto, partículas de caucho, neumáticos reciclados, propiedades mecánicas, carrocería.

\section{Introducción.}

La generación de neumáticos de desecho en todo el mundo es de aproximadamente un millón de dólares por año y se espera que ésta cifra incremente en el futuro (Abu-Jdayil, Mourad, \& Hussain, 2016). Según el Ministerio del Ambiente (MAE), en el Ecuador se 
desecha anualmente millones de neumáticos, de los cuales un bajo porcentaje de estos son reutilizados para el reencauche, pero la gran mayoría son incinerados o depositados en basureros al aire libre, lo que presenta una amenaza contra el medio ambiente (Ministerio del Ambiente, 2013).

Actualmente la tasa de reutilización de neumáticos de desecho es mucho menor que el producido, se prevé para el 2018 la demanda mundial de neumáticos reciclados crecerá un $2,4 \%$ que representa 13,34 millones de toneladas mientras que el consumo total del caucho aumentará a una tasa de 3,3\% equivalente a 29,37 millones de toneladas con respecto al año anterior (International Rubber Study Group, 2017).

Una de las aplicaciones de caucho reciclado con potencial en el futuro es la incorporación del caucho particulado en matrices poliméricas para la obtención de materiales compuestos, los cuales son muy apetecidos en la industria actual (Peláez, Velásquez, \& Giraldo, 2017). Las industrias manufactureras de índole estructural y automotriz buscan la aplicación de materiales amigables con el ambiente con propiedades mecánicas que satisfagan los esfuerzos sometidos a sus productos finales (Paredes, Pérez, \& Castro, 2017).

La adición de partículas de caucho de neumáticos reciclados, en polímeros termorrígidos o termoplásticos ha sido el foco de la investigación en esta área. (Silva et al., 2012), promueven el reciclaje de neumáticos de desecho mediante la incorporación de los residuos de caucho en una matriz polimérica, creando un material sostenible que cumpla con los estándares y normas técnicas de la industria brasileña e internacionales.

Su estudio se basó sobre el efecto de las propiedades físicas y mecánicas de los materiales compuestos de matriz polimérica de resina epoxi con y sin adición de anhídrido maléfico ( $1.5 \%$ en masa) y fracciones en masa de las partículas para estudio fueron al $10 \%, 25 \%$ y $50 \%$. Uno de los resultados más significativos del estudio fue la composición de $25 \%$ en masa de partículas de caucho de tamaño 100/200 US-Tyler, mostrando aplicaciones prometedoras en ingeniería además de un porcentaje significativo de reutilización de residuos de caucho en la producción de este material.

(Olmos, Vela, Alvarez, \& González, 2013), utilizaron partículas de caucho de los neumáticos en desuso para modificar una base epoxi termoendurecible con el objetivo de estudiar el comportamiento mecánico del material. El contenido de partículas de caucho para los especímenes de estudio fue de $0 \%, 1 \%, 2 \%, 4 \%$ y $10 \%$. Los resultados obtenidos relacionados con el contenido de partícula fue, que para concentraciones menores al $4 \%$, el comportamiento en condiciones más severas (ensayo Charpy) se mantiene o aumenta ligeramente la energía absorbida por el material, mientras que la fuerza máxima aplicada y la energía total absorbida en la ruptura aumentan cuando disminuye el tamaño de la partícula; tales resultados evidencia que el tamaño de las partículas es un factor importante para el rendimiento final de los materiales bajo estudio. 
Ciertas investigaciones han establecido que la adición de partículas en materiales termoplásticos lleva a la fragilidad del compuesto debido a la formación y propagación de un cuello en el polímero matriz sometido a tensión (Serenko, Avinkin, \& Bazhenov, 2002).

(Abu-Jdayil et al., 2016), formularon y desarrollaron un compuesto de poliéster insaturado con caucho como material aislante con 8 diferentes concentraciones en volumen de caucho $(5 \%, 10 \%, 15 \%, 20 \%, 25 \%, 30 \%, 35 \%$ y $40 \%)$ cuyos resultados mostraron que la mezcla de partículas de caucho con la matriz poliéster disminuyó tanto la resistencia a la compresión como a la tensión y módulos del compuesto. Además, a medida que aumenta el contenido de caucho reduce notablemente las resistencias del compuesto, y mientras el tamaño de la partícula sea menor, menor será su resistencia y módulo de elasticidad.

(Valášek, Müller, \& Ružbarský, 2014), en su estudio corroboraron la hipótesis de obras de otros autores, la presencia de partículas inorgánicas tiene un impacto negativo con respecto a las propiedades mecánicas del material, sin embargo, en las pruebas realizadas evidenciaron que mientras aumenta la concentración de partículas de caucho reciclado lleva a disminuir proporcionalmente valores de dureza, resistencia a la tracción y cizalladura, pero pese a los resultados obtenidos establecen que es necesario preferir el uso de materiales de desecho debido a que es un tipo de reciclaje rentable y respetuoso con el medio ambiente adecuado para aplicaciones donde no se requiere valores altos de resistencia.

La industria automotriz y carrocera tiene el reto de reemplazar las partes externas de los vehículos por materiales biodegradables, reciclables o al menos incinerables para transformarles en alguna fuente de energía como la térmica, con el propósito de preservar el medio ambiente y el planeta. El reemplazo de componentes exteriores es más complicado ya que estas partes no están protegidas de las condiciones climáticas, por lo que una alternativa sería obtener un material compuesto con matriz polimérica reforzado con partículas de neumáticos desechados, para la construcción de partes exteriores de carrocerías y que resistan las inclemencias del medio ambiente, y una vez cumplida su vida útil podamos incinerarlos y transformarlos en energía térmica.

\section{Materiales y métodos.}

Para iniciar el estudio se elaboraron probetas del material compuesto mediante el método de estratificación manual a compresión atornillada utilizando moldes metálicos con distintos porcentajes de partículas de caucho de neumáticos reciclados de tres granulometrías diferentes G1 $(0,05 \mathrm{~mm}-0,6 \mathrm{~mm}), \mathrm{G} 2(0,6-1,18 \mathrm{~mm})$ y G3 $(1,18 \mathrm{~mm}-$ 2,36mm) para posteriormente realizar ensayos de tracción, flexión y compresión, bajo normas ASTM D3039-14 (ASTM D3039/D3039M-14, 2014), ASTM D7264-15 (ASTM D7264/D7264M-15, 2015) y ASTM D695-15 (ASTM D695-15, 2015) respectivamente, se preparó el molde metálico limpiándole y untándole de cera desmoldante marca AUTO 
CARE, se añadió el 10\% de estireno monómero a la resina poliester ortoftálica de propósito general marca andercol POL R 33000 al $70 \%$ en sólidos más el $0.5 \%$ en peso de octoato de cobalto al $12 \%$ de Co y $2 \%$ en peso de MEK peróxido metil etil cetona al $9 \%$ de oxígeno activo, las partículas de caucho se añadieron a la mezcla variando el porcentaje de refuerzo en $10 \%, 20 \%, 30 \%, 35 \%$ y $40 \%$ para ser coladas en el molde metálico y ser cortadas con cortadora laser después de 21 días de curado con las dimensiones que especifica las normas ASTM, como se observa en las figuras 1,2 y 3.

Figura 1. Dimensiones en mm de la probeta para ensayo a tracción según norma ASTM D3039-14 donde $e$ es el espesor.

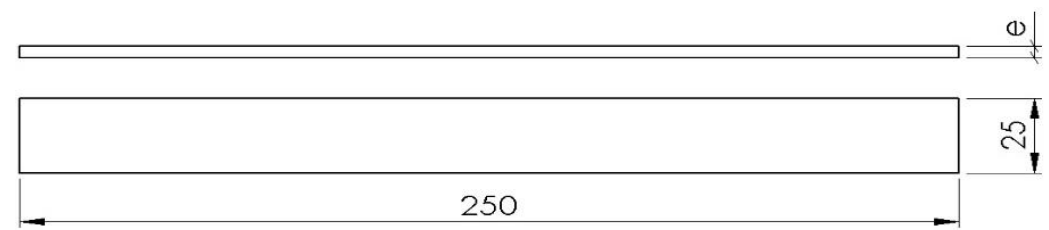

Fuente: Grupo de investigación.

Figura 2. Dimensiones en $\mathrm{mm}$ de la probeta para ensayo a flexión según norma ASTM D 7264-15 donde $e$ es el espesor.

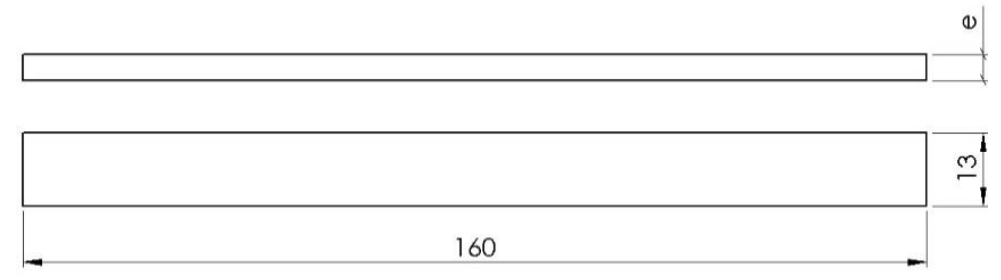

Fuente: Grupo de investigación.

Figura 3. Dimensiones en mm de la probeta para ensayo a compresión según norma ASTM D 695-15.
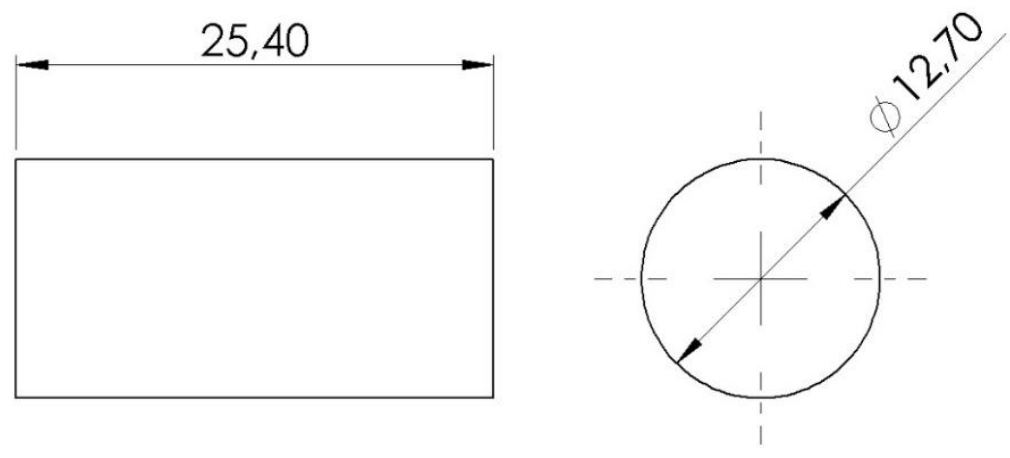

Fuente: Grupo de investigación. 
Para el ensayo de tracción, flexión y compresión se utilizó la máquina universal para polímeros Metrotest modelo $50 \mathrm{KN}$ serie 8210M002 del laboratorio de resistencia de materiales del Centro de Fomento Productivo Metalmecánico Carrocero de Tungurahua, que se muestra en la figura 4.

Figura 4. Máquina Universal Metrotest modelo $50 \mathrm{KN}$ serie 8210M002.

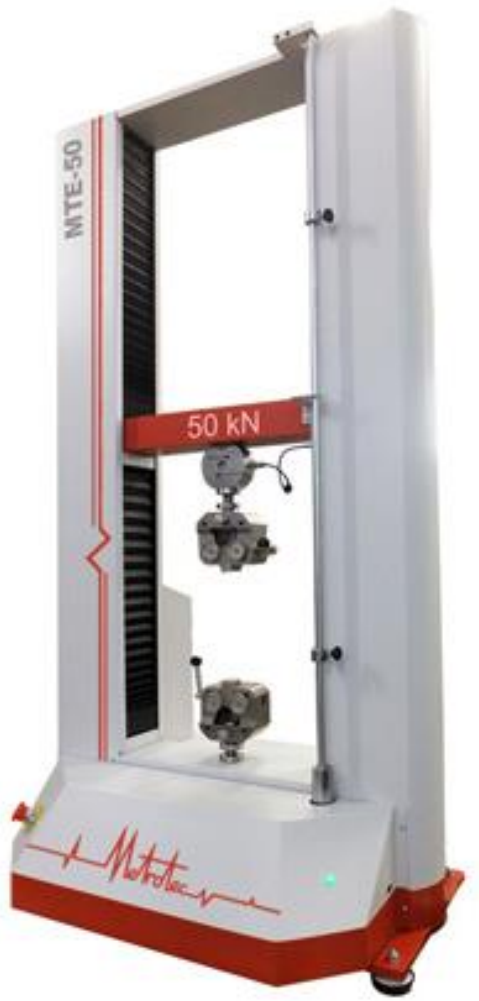

Fuente: Catálogo Metrotest

Para analizar la estructura interna del material compuesto se utilizó un microscopio electrónico de barrido TESCAN modelo VEGA 3 SBU, del laboratorio de materiales de la Carrera de Ingeniería Mecánica de la Universidad Técnica de Ambato.

\section{Resultados.}

Una vez obtenidas las probetas del material compuesto de matriz polimérica reforzado con partículas de caucho de neumáticos reciclados con las tres granulometrías (G1, G2 y G3), y variando la fracción volumétrica del material de refuerzo $(0 \%, 10 \%, 20 \%, 30 \%, 35 \%$ y $40 \%$ ) se procedió a ensayarlas mecánicamente a tracción, flexión y compresión, obteniendo los resultados que se evidencian a continuación: 


\section{Resultados del ensayo a tracción.}

En la tabla 1, figura 5 y figura 6, se muestra los resultados promedio obtenidos del ensayo a tracción para las tres granulometrías (G1, G2 y G3), utilizando la norma ASTM D3039-14.

Tabla 1. Resultados del ensayo de tracción.

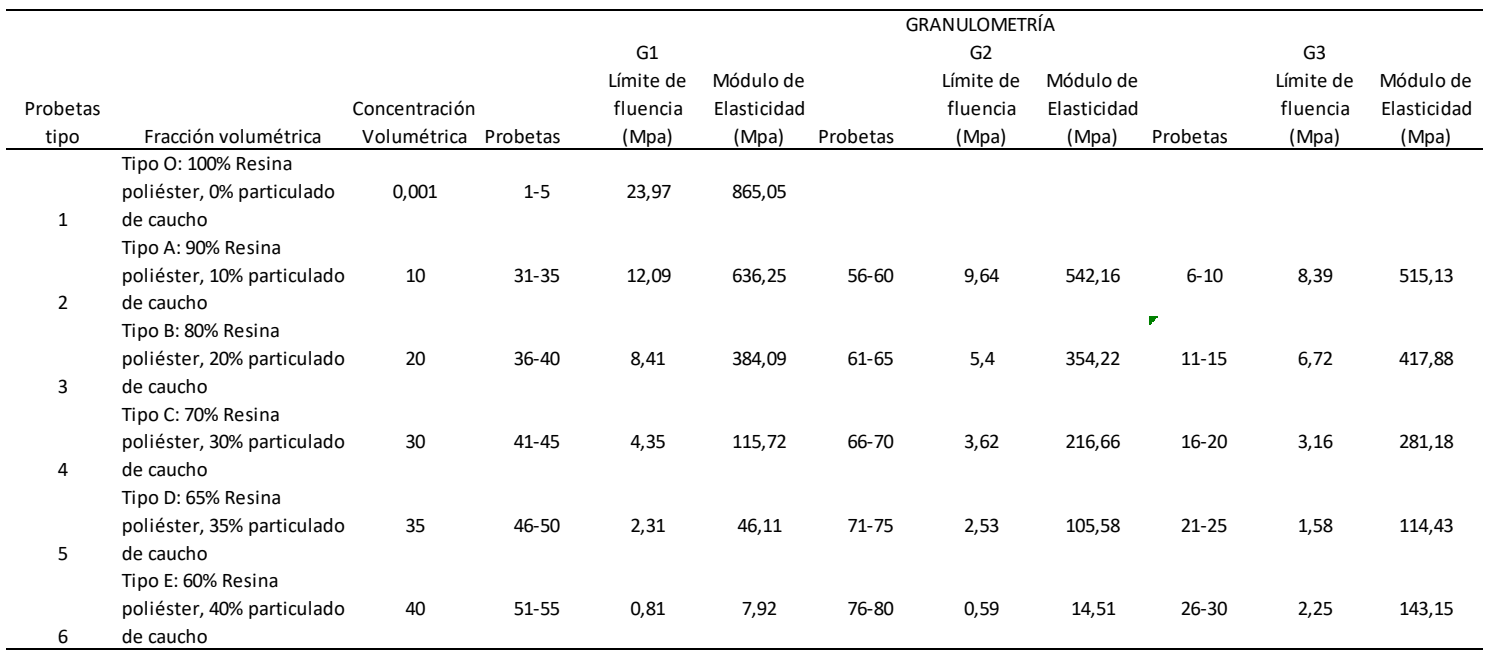

Fuente: Grupo de investigación.

Figura 5. Curvas fluencia a tracción Vs concentración volumétrica.

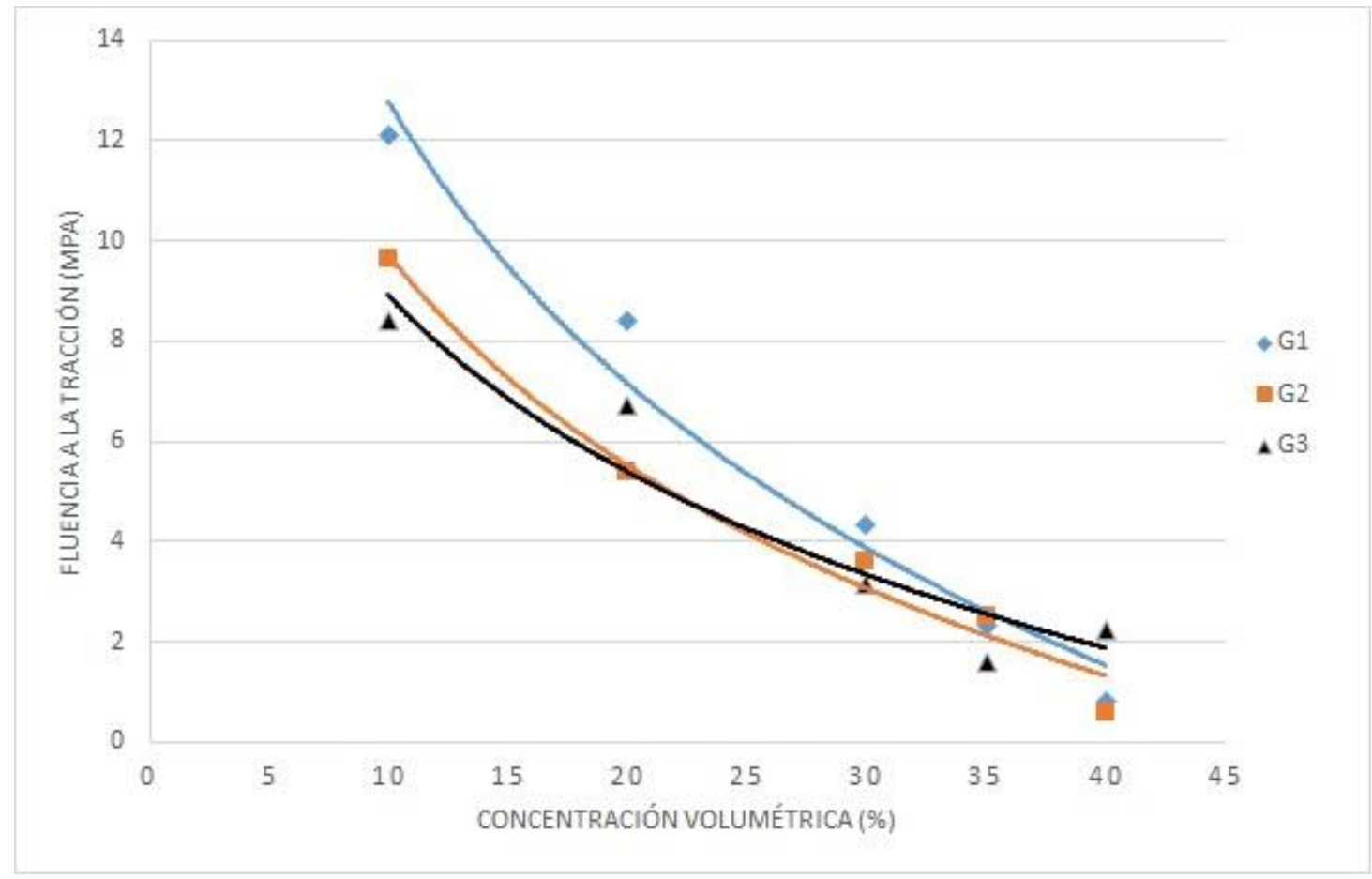

Fuente: Grupo de investigación. 
En la figura 5, se puede observar que el límite de fluencia del material sometido a tracción, en primer lugar, disminuye al aumentar la concentración volumétrica y en segundo lugar para concentraciones de alrededor del $10 \%$ es mayor con partículas de caucho cuya granulometría es la G1 y disminuye progresivamente a medida que la granulometría aumenta a G2 y G3 respectivamente. Además, se puede apreciar que para concentraciones del $20 \%$ se tiene límites de fluencia similares para las granulometrías G2 y G3, y por último se observa que a medida que la concentración aumenta a valores de entre $35 \%$ y $40 \%$ el límite de fluencia es similar con los tres tipos de granulometrías ensayados.

Figura 6. Curvas módulo de elasticidad Vs Concentración Volumétrica.

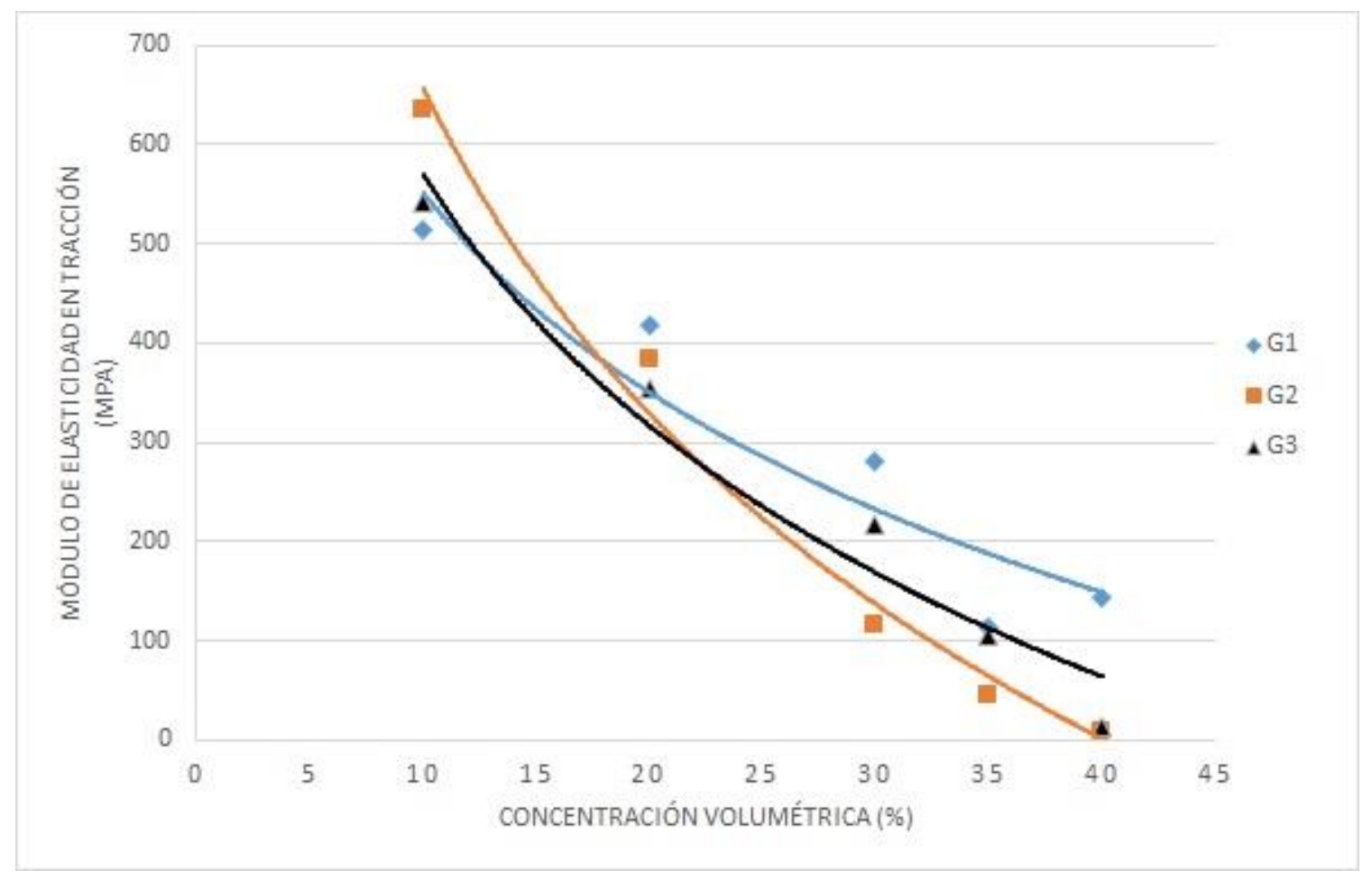

Fuente: Grupo de investigación.

En la figura 6, se observan los resultados de ensayos a tracción en los que se puede observar en primer lugar que el módulo de elasticidad disminuye al aumentar la concentración volumétrica, y en segundo lugar a bajas concentraciones no existe una influencia muy marcada al variar la granulometría, cuando las concentraciones son alrededor del $20 \%$. 


\section{Resultados del ensayo a flexión.}

En la tabla 2, figura 7 y figura 8, se muestra los resultados promedios obtenidos del ensayo a tracción para las tres granulometrías (G1, G2 y G3), utilizando la norma ASTM D726415 .

Tabla 2. Resultados del ensayo a flexión.

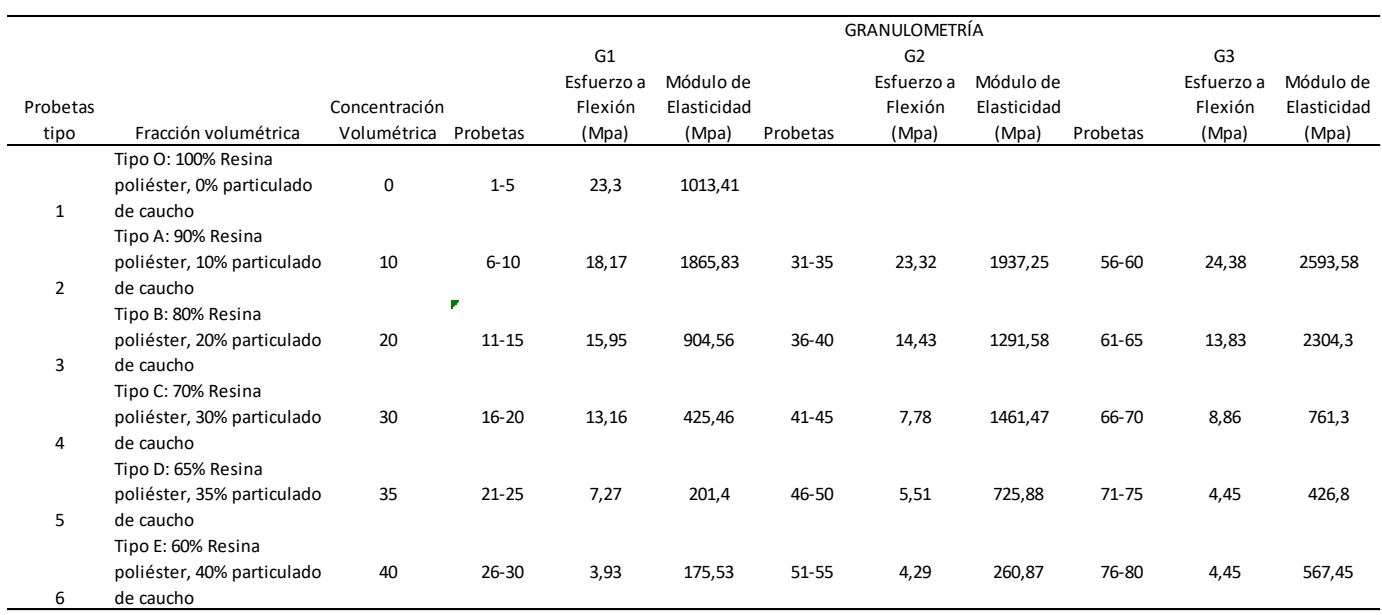

Fuente: Grupo de investigación.

Figura 7. Curvas fluencia a flexión Vs concentración volumétrica.

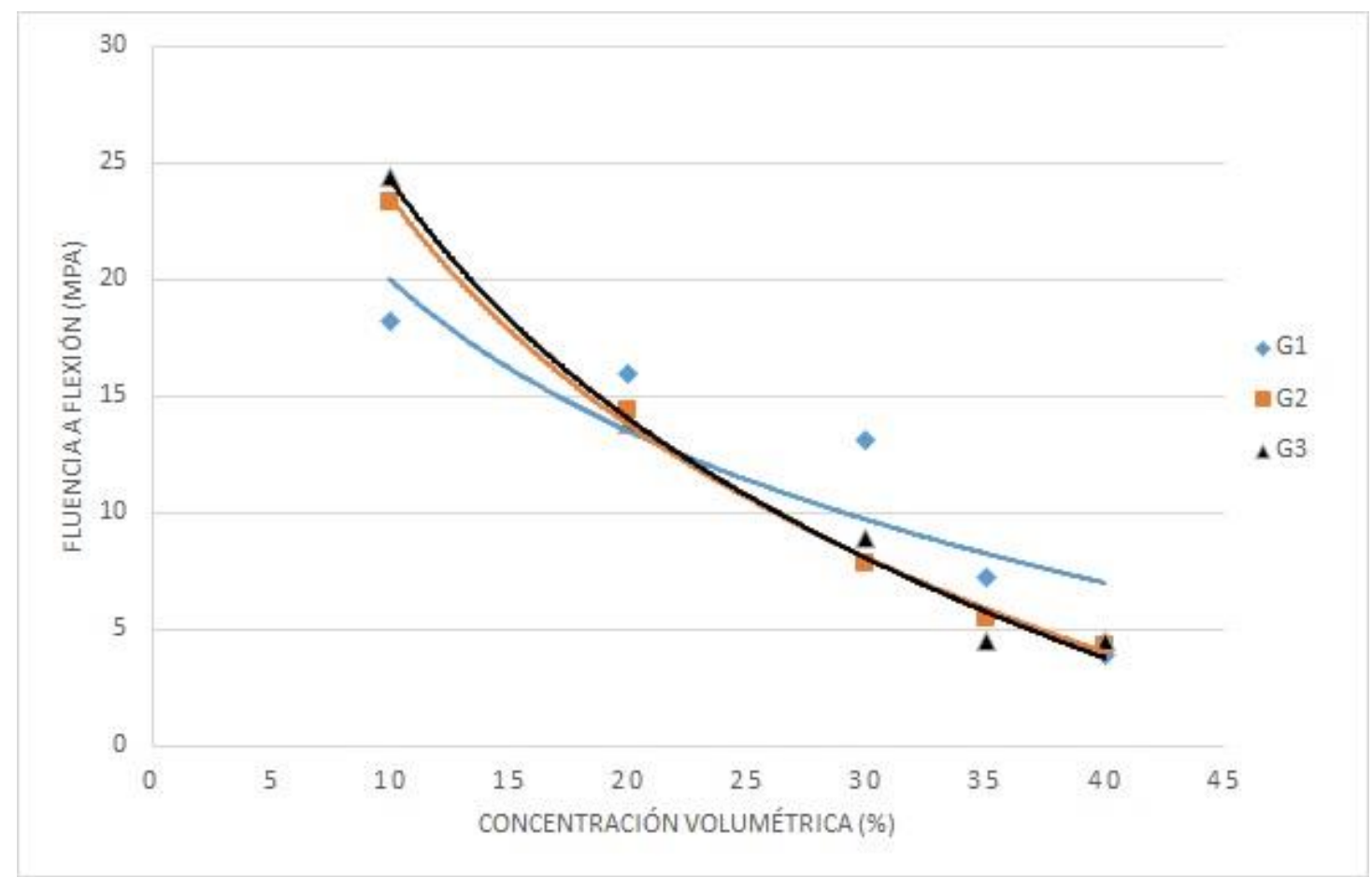

Fuente: Grupo de investigación. 
En la figura 7, se representa la relación que existe entre el límite de fluencia a flexión y concentración volumétrica y granulometría, de la cual se observa básicamente que el límite de fluencia a flexión disminuye a medida que la concentración aumenta, sin embargo, la variación de la granulometría no influye en el mismo.

Figura 8. Curvas módulo de elasticidad Vs Concentración Volumétrica.

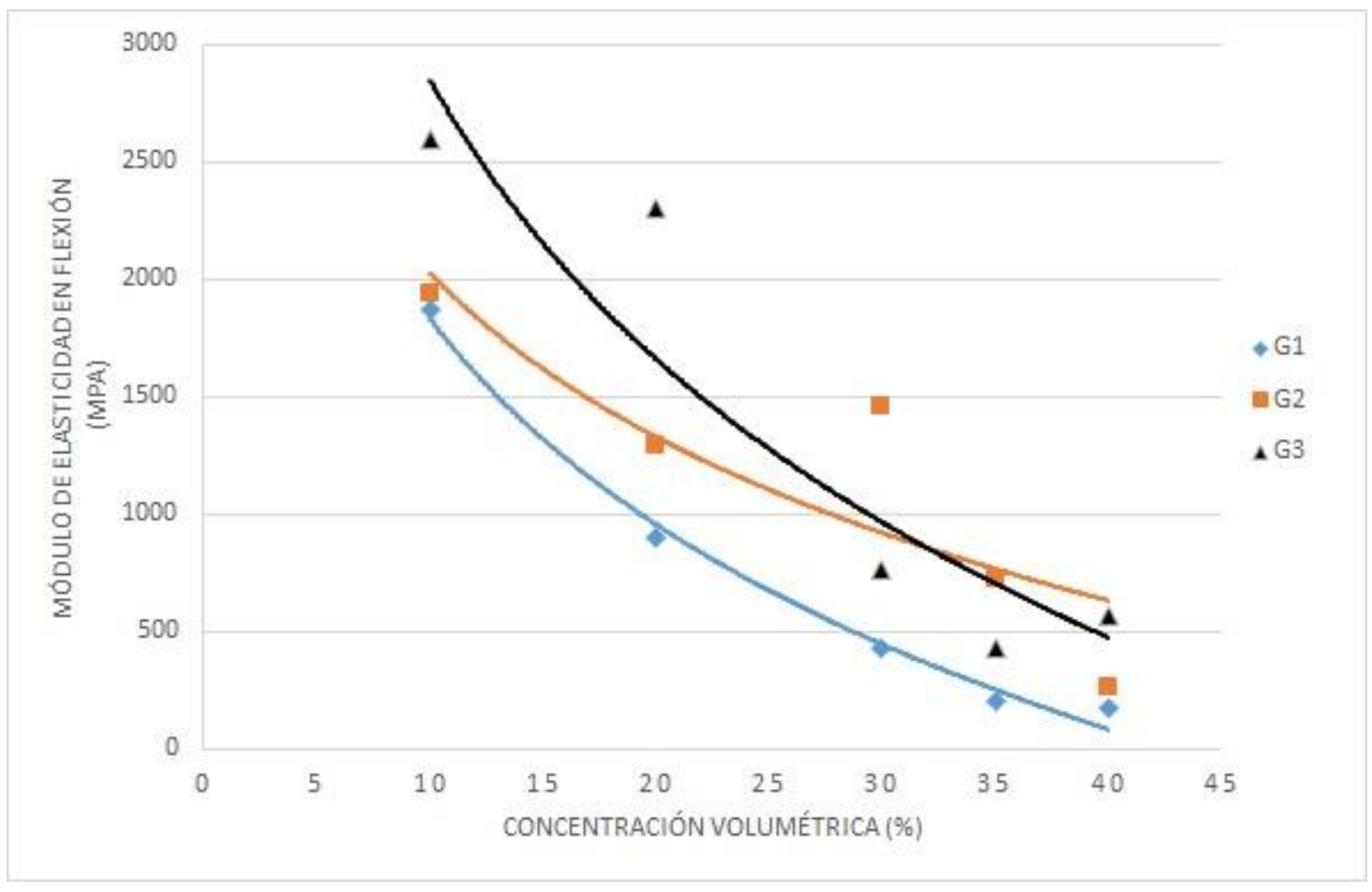

Fuente: Grupo de investigación.

En la figura 8, se presenta, en primer lugar, la relación que existe entre el módulo de elasticidad y la concentración volumétrica de probetas ensayadas a flexión se puede apreciar que a medida que aumenta dicha concentración el módulo disminuye. Además, existe una tendencia a disminuir a medida que aumenta la granulometría, sin embargo, para granulometrías G1 y G2 con concentraciones de alrededor al $35 \%$ el módulo de elasticidad es similar.

\section{Resultados del ensayo a compresión.}

En el ensayo a compresión las probetas fueron colocadas entre dos plataformas de la máquina, la carga fue aplicada a $100 \mathrm{~mm}$ de desplazamiento y a una velocidad de deformación constante de $5 \mathrm{~mm} / \mathrm{min}$, aplicando la norma ASTM D695-15. En la tabla 3, se muestran los resultados de este ensayo para cada concentración volumétrica y granulometría. 
Tabla 3. Resultados del ensayo a compresión.

\begin{tabular}{|c|c|c|c|c|c|c|c|c|}
\hline \multirow{4}{*}{$\begin{array}{c}\text { Probetas } \\
\text { tipo }\end{array}$} & \multirow[b]{4}{*}{ Fracción volumétrica } & \multirow{3}{*}{\multicolumn{2}{|c|}{ Concentración }} & & & & & \\
\hline & & & & \multirow{3}{*}{$\begin{array}{c}\text { G1 } \\
\text { Esfuerzo a } \\
\text { compresión }\end{array}$} & & G2 & & \multirow{3}{*}{$\begin{array}{c}\text { G3 } \\
\text { Esfuerzo a } \\
\text { compresión }\end{array}$} \\
\hline & & & & & & Esfuerzo a & & \\
\hline & & Volumétrica & Probetas & & Probetas & compresión & Probetas & \\
\hline & Tipo A: 90\% Resina & & & & & & & \\
\hline & poliéster, $10 \%$ particulado & 10 & $6-10$ & 34,94 & $31-35$ & 42,97 & $56-60$ & 31,75 \\
\hline \multirow[t]{3}{*}{1} & de caucho & & & & & & & \\
\hline & Tipo B: 80\% Resina & & & & & & & \\
\hline & poliéster, $20 \%$ particulado & 20 & $11-15$ & 25,95 & $36-40$ & 20,61 & $61-65$ & 27,32 \\
\hline \multirow[t]{3}{*}{2} & de caucho & & & & & & & \\
\hline & Tipo C: 70\% Resina & & & & & & & \\
\hline & poliéster, $30 \%$ particulado & 30 & $16-20$ & 7,49 & 41-45 & 10,83 & $66-70$ & 9,89 \\
\hline \multirow[t]{3}{*}{3} & de caucho & & & & & & & \\
\hline & Tipo D: 65\% Resina & & & & & & & \\
\hline & poliéster, $35 \%$ particulado & 35 & $21-25$ & 14,32 & $46-50$ & 7,21 & $71-75$ & 8,87 \\
\hline \multirow[t]{3}{*}{4} & de caucho & & & & & & & \\
\hline & Tipo E: 60\% Resina & & & & & & & \\
\hline & poliéster, $40 \%$ particulado & 40 & $26-30$ & 9,61 & $51-55$ & 8,42 & $76-80$ & 8,34 \\
\hline 5 & de caucho & & & & & & & \\
\hline
\end{tabular}

Fuente: Grupo de investigación.

Figura 9. Curvas esfuerzo a compresión Vs concentración volumétrica.

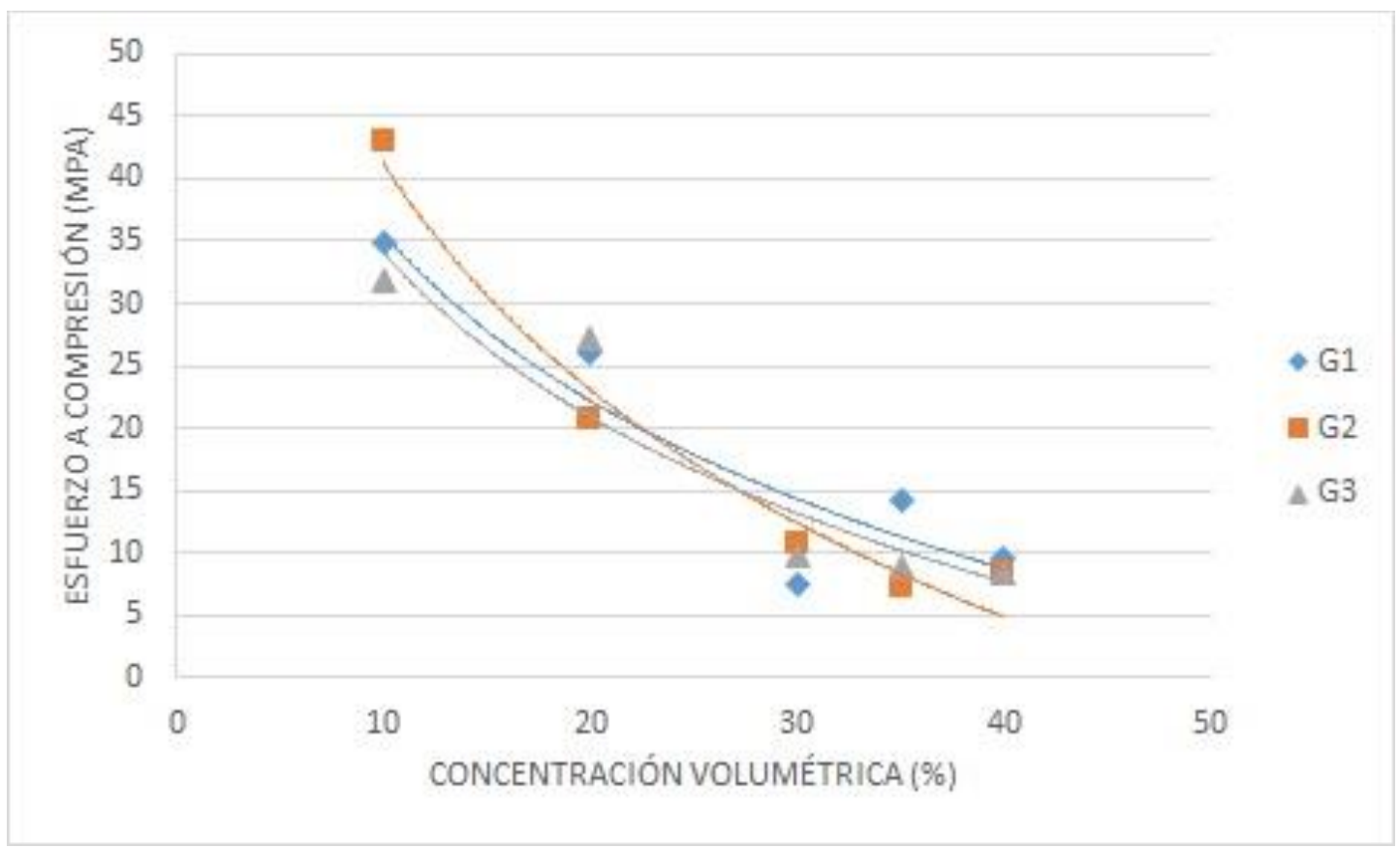

Fuente: Grupo de investigación.

En la figura 9, se puede observar que la resistencia a compresión del material compuesto, en primer lugar, disminuye al aumentar la concentración volumétrica y en segundo lugar, no se observan cambios significativos al cambiar la granulometría. 


\section{Resultados de la microscopía electrónica de Barrido (SEM).}

Al realizar el análisis interno del material compuesto mediante microscopia electrónica de barrido (SEM) de tres fracciones volumétricas $(10 \%, 20 \%$ y 30\%), presentó la morfología que se muestra en la figura 10.

Figura 10. Microscopía electrónica de barrido del material compuesto, a) fracción volumétrica de $10 \%$ de refuerzo, b) fracción volumétrica de $20 \%$ de refuerzo, c) fracción volumétrica de 30\% de refuerzo.
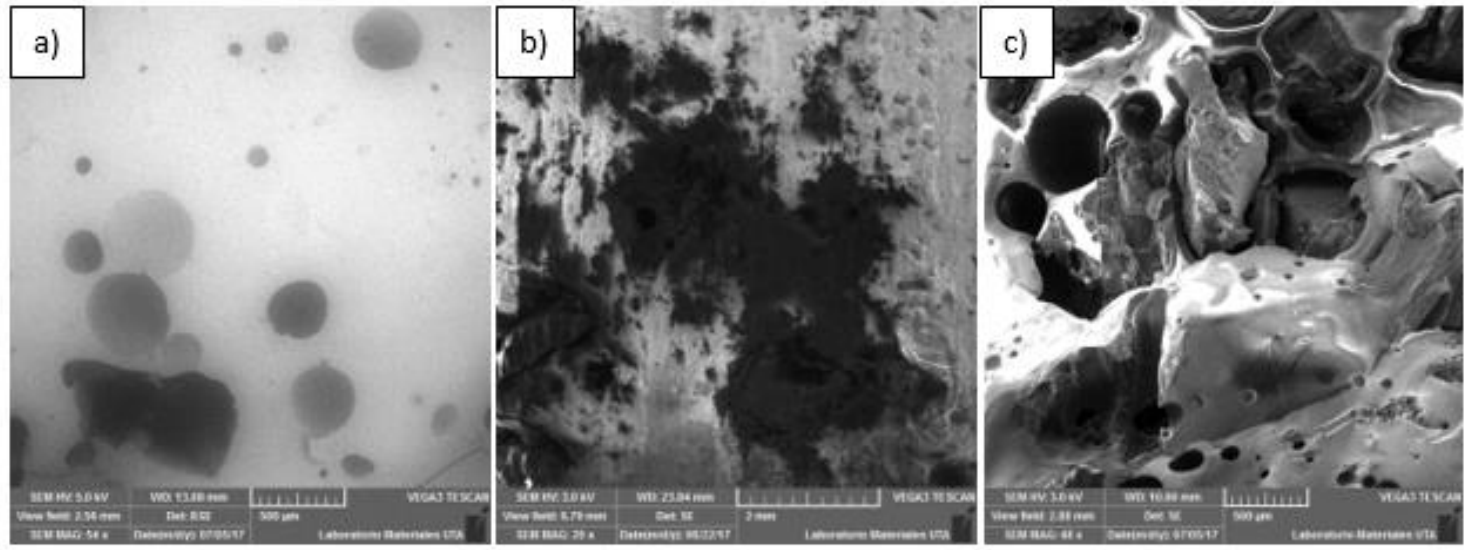

Fuente: Grupo de investigación.

Las micrografías evidencian que el material compuesto en los tres casos presenta una distribución normal del particulado de caucho con una excelente adherencia a la matriz (resina poliéster).

\section{Conclusiones.}

- Se ha investigado el comportamiento mecánico del material compuesto de resina poliéster con partículas de caucho de neumáticos reciclados con tres tamaños de partículas y cinco concentraciones volumétricas de contenido de caucho. Los resultados evidencian que tanto el esfuerzo a tracción, flexión y compresión de los materiales compuestos disminuyen al aumentar el contenido de partículas de caucho. El módulo de elasticidad a tracción tiende a disminuir al aumentar el contenido de las partículas y al disminuir su tamaño, mientras que el módulo de elasticidad a flexión en la concentración volumétrica al $10 \%$ de partículas de caucho en la que se evidencia un incremento del $84,11 \%$ para G1; $91,16 \%$ para G2 y $155,93 \%$ para G3, comparado con la resina poliéster pura (sin refuerzo), por lo tanto, también se determina que incrementa su módulo de elasticidad a flexión al incrementar el tamaño de partícula.

- Con las propiedades mecánicas obtenidas mediante los ensayos, se determina que el material compuesto con granulometría G3 es apto para trabajar a flexión ya que 
incrementa su módulo de elasticidad convirtiéndole en un material elástico, por lo que se aplicará en partes exteriores de carrocerías como en guardafangos, guardapolvos, en bases entre chasis y carrocería para absorber vibraciones, en defensas de guardachoques delantero y posterior para absorber impactos, en masacrillas y como material aislante del ruido, calor y electricidad.

\section{Agradecimiento.}

A la Dirección de Investigación y Desarrollo de la Universidad Técnica de Ambato por el financiamiento del proyecto de investigación "Aplicabilidad de un material compuesto con matriz polimérica reforzado con partículas de neumáticos reciclados en partes exteriores de carrocerías de la industria carrocera de Tungurahua” del que es parte esta investigación.

Al Centro de Investigación y Desarrollo de la Fuerza Aérea Ecuatoriana y Carrocerías Santacruz, por su apoyo incondicional al desarrollo del proyecto.

\section{Referencias bibliográficas.}

Abu-Jdayil, B., Mourad, A. H. I., \& Hussain, A. (2016). Investigation on the mechanical behavior of polyester-scrap tire composites. Construction and Building Materials, 127, 896-903.

https://doi.org/10.1016/j.conbuildmat.2016.09.138

ASTM D3039/D3039M-14. (2014). Standard Test Method for Tensile Properties of Polymer Matrix Composite Materials. ASTM International. West Conshohocken, PA. https://doi.org/10.1520/D7264_D7264M-14

ASTM D695-15. (2015). Standard Test Method for Compressive Properties of Rigid Plastics. ASTM International (Vol. i). West Conshohocken, PA. https://doi.org/10.1520/D0695-15.2

ASTM D7264/D7264M-15. (2015). Standard Test Method for Flexural Properties of Polymer Matrix Composite Materials. ASTM International. West Conshohocken, PA. https://doi.org/10.1520/D7264_D7264M-15

International Rubber Study Group. (2017). Latest World Rubber Industry Outlook now available from IRSG. Retrieved from http://www.rubberstudy.com/newsarticle. $\operatorname{aspx} ? \mathrm{id}=5135 \& \mathrm{~b}=$ default.aspx

Ministerio del Ambiente. (2013). La Gestión Integral de Neumáticos Usados optimiza recursos para el manejo seguro de desechos. Retrieved from http://www.ambiente.gob.ec/ 
Olmos, D., Vela, R., Alvarez, A., \& González, J. (2013). Rubber particles from tires out of use as toughness modifiers of epoxy-based thermosets. Journal of Adhesion, 89(9), 697-713.

https://doi.org/10.1080/00218464.2012.752327

Paredes, J. G., Pérez, C. F., \& Castro, C. B. (2017). Análisis de las propiedades mecánicas del compuesto de matriz poliéster reforzado con fibra de vidrio 375 y cabuya aplicado a la industria automotriz ( Analysis of the mechanical properties of the composite of polyester matrix reinforced with glass fiber, 1-15.

Peláez, G. J., Velásquez, S. M., \& Giraldo, D. H. (2017). Aplicaciones de caucho reciclado: Una revisión de la literatura. Ciencia E Ingeniería Neogranadina, 27(2), 27-50. https://doi.org/10.18359/RCIN.2143

Serenko, O. A., Avinkin, V. S., \& Bazhenov, S. L. (2002). Fracture of a composite material based on a uniformly deformable polymeric matrix and rubber particles. Doklady Physics, 47(1), 72-73. https://doi.org/10.1134/1.1450666

Silva, A. S., Nacif, G. C. L., Panzera, T. H., Christoforo, A. L., Batista, F. B., \& Mano, V. (2012). Incorporação de resíduos de borracha em compósitos de matriz polimérica termorrígida. Revista Materia, 17(4), 1158-1165. https://doi.org/10.1590/S151770762012000400006

Valášek, P., Müller, M., \& Ružbarský, J. (2014). Using Recycled Rubber Particles as Filler of Polymers. Applied Mechanics and Materials, 616, 260-267. https://doi.org/10.4028/www.scientific.net/AMM.616.260

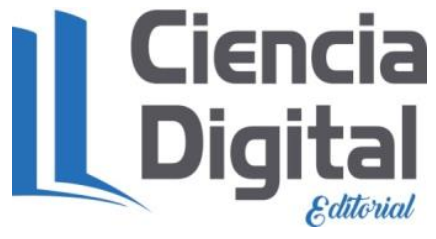




\section{Para citar el artículo indexado.}

Espín S., Guamanquispe J., Acosta E., Jara A., León G. \& Sevilla M. (2018). Caracterización del material compuesto de resina poliester con partículas de caucho reciclado y su aplicabilidad en carrocerías. Revista electrónica Ciencia Digital 2(2), 48-63. Recuperado desde:

http://cienciadigital.org/revistacienciadigital2/index.php/CienciaDigital/article/view/72/67

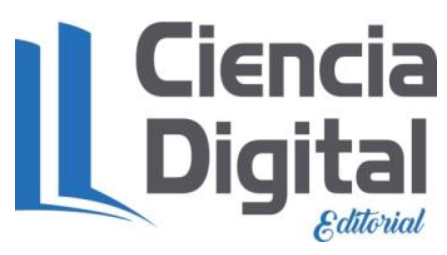

El artículo que se publica es de exclusiva responsabilidad de los autores y no necesariamente reflejan el pensamiento de la Revista Ciencia Digital.

El articulo queda en propiedad de la revista y, por tanto, su publicación parcial y/o total en otro medio tiene que ser autorizado por el director de la Revista Ciencia Digital.
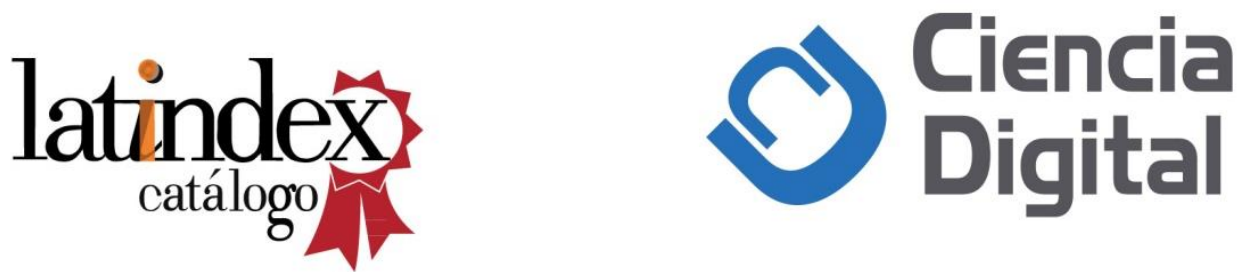